\title{
Editorial
}

\section{Personal Assistance and Monitoring Devices Applications}

\author{
Renato Ferrero $\mathbb{D}$, ${ }^{1}$ Maurizio Rebaudengo $\mathbb{D}^{1},{ }^{1}$ and Francisca Rosique $\mathbb{C}^{2}$ \\ ${ }^{1}$ Dipartimento di Automatica e Informatica, Politecnico di Torino, 10129 Torino, Italy \\ ${ }^{2}$ Universidad Politécnica de Cartagena, Cartagena, Spain \\ Correspondence should be addressed to Renato Ferrero; renato.ferrero@polito.it
}

Received 3 June 2019; Accepted 3 June 2019; Published 7 July 2019

Copyright (c) 2019 Renato Ferrero et al. This is an open access article distributed under the Creative Commons Attribution License, which permits unrestricted use, distribution, and reproduction in any medium, provided the original work is properly cited.

During almost the whole day, people are carrying or wearing one or more personal devices, such as smartphone, smartwatch, smartband, activity tracker, or smart ring. From one side, these devices provide an extraordinary source of information. They can collect health parameters, such as heart rate and blood pressure, and measure the activity of the user, for example, by counting the number of steps, track position, and speed of the user. From the other side, the personal devices offer an intuitive and implicit interaction with the user. Their interface is designed to recognize the user's actions and respond to them. A natural communication, based on touch, gestures, voice, and sounds, is established with the user in order to recognize his/her actions and respond to them. Furthermore, the personal devices can be aware of the usage context, for example, by automatically recognizing the activity performed by the user. Their behaviour can be proactive: by recording previous routine and needs of the user, they can propose specific suggestions related to the current context, so anticipating the explicit user input. New applications of the personal devices are arising in many fields, such as health, wellness, and entertainment. At the same time, the service can be personalized and adaptive, because the continuous flow of collected data simplifies the knowledge of the user and the understanding of routine changes.

This special issue contains excellent contributions about emerging applications of personal devices with intuitive user interaction. In addition, a comprehensive review paper gives an insight into a healthcare application of personal devices. In this way, we think that the special issue may attract a broad section of readership, from beginners to advanced researchers.
A common application in mobile healthcare is fall avoidance, due to the high social cost caused by unintentional falls. Personal devices have been exploited in many fall detection systems, which are able to recognize falls and notify user's acquaintances in order to provide quick assistance to the user after the fall. However, if the goal is the fall avoidance, more promising approaches require the evaluation of the fall risk. The prediction of the fall in real time is used to alert the user before the fall occurrence or to activate some external aid, such as a walking assist robot, whereas proper exercise for improving gait and mobility can be assigned to the user according to the assessment of his/her probability of future falls. The paper of M. Hemmatpour et al. provides a detailed review on fall prediction and prevention systems based on wearable devices. The posture and gait of the user are commonly studied by means of accelerometer, gyroscope, motion sensor, and piezoelectric sensor. The main features extracted from the collected data are gait speed and acceleration, trunk tilt, foot posture, and duration of the transitions between postures. Different machine learning algorithms can be applied to process these features: threshold-based algorithm, decision tree, support vector machine, and fuzzy logic. In the paper of M. Hemmatpour et al., a dataset of walk patterns was populated by recording data when a group of users is asked to walk in a flat area, where different types of obstacles cause irregular gaits. The level of fall risk predicted by the different machine learning algorithms was compared by using the speed, acceleration, and tilt extracted from the collected data. The evaluation criteria for the outcome of the machine learning algorithms include specificity, sensitivity, accuracy, error rate, precision, and recall. According to the analysis, normal and abnormal walk patterns can be 
distinguished at best by evaluating the tilt feature with a decision tree.

Another healthcare application of wearable devices is the monitoring and controlling of heart problems, as shown in the paper of A. J. A. Majumder et al. The authors have developed a smart IoT system composed of embedded temperature and pulse sensors for data collection, an Arduino board for analog-to-digital converter, a Bluetooth chip for data transmission, and a smartphone for data analysis and visualization. Temperature and electrocardiogram (ECG) data are read from the sensors at $5 \mathrm{~Hz}$ and $160 \mathrm{~Hz}$, respectively. First, a filtering technique is applied to the ECG data to reduce the noise, with removal of baseline wander and high-frequency component. Then, three features are extracted from the ECG signal: heart rate, that is, number of beats per minute; R$\mathrm{R}$ interval, that is, interval between successive heartbeats; and ST segment, that is, length of the flat section between the waves in a heartbeat. A novel algorithm based on the decision tree model with a standard deviation statistical analysis has been implemented in order to evaluate the three ECG features and the temperature. Input data are analysed every second; the algorithm evaluates the analysis results in the last five seconds to determine the degree of heart rate abnormality, according to five warning levels. The developed IoT system has been evaluated with healthy test subjects, without triggering any warnings and with data from an online database of patients who suffered from sudden cardiac deaths. In the latter case, the algorithm is able to distinguish the abnormal ECG pattern and raise the proper warning level.

Wearable technology can be a reliable and objective support for humans' senses. An example is provided in the paper of V. Ferraro et al. about a plurisensorial device aiming at preventing occupational disease. In particular, a prototype was designed for coating plants, where the concentration of Volatile Organic Compounds (VOC) is high during material lacquering. VOC-prolonged inhalation may cause asthma, lung cancer, pulmonary and respiratory diseases, and so forth, so environmental monitoring and personal protective equipment are mandatory by law. However, according to the authors' initial observation, the lacquering tasks were performed in cabins equipped with an aspiration system to reduce the inner air pollution, but the workers did not wear the protective mask. In fact, despite the strong smell of VOC, the workers' perception about them was low: this is an adaptation due to the long-term exposure. Without feeling bad odours, the workers do not perceive the dangerousness of VOC. V. Ferraro et al. have designed and prototyped a wearable system able to provide objective data to the users in order to enhance their perception of risk. The system is composed of an electronic nose device, a smart protective mask, a chest band, and a mobile application for smartphone. The electronic nose device is equipped with a VOC sensor and gives feedback about the air quality thanks to coloured LEDs. The mask monitors the user's breath by means of temperature and humidity sensors. The chest band measures the respiration frequency. Data collected by the three devices are sent via Bluetooth to the mobile application, which provides real time and historical statistics. The testing of the prototype reveals that, during the tasks performed in the cabin, the level of contaminants was high during most of the time. According to the final interview, the workers changed their consciousness about their health conditions and were interested in wearing the protective mask.

T. Khan et al. present a study of the impact of an Augmented Reality (AR) mobile application on learning motivation of a group of undergraduate health science students. They present a very complete literature review, emphasizing the advantages and challenges of the current state of augmented reality technology in education. Preusage and postusage questionnaires were used as instruments for data collection. To measure motivation, authors use the Attention, Relevance, Confidence, and Satisfaction (ARCS) model. Based on the ARCS factors, the authors developed a questionnaire in the form of a five-point Likert scale. The results showed that using an augmented reality mobile application can increase the learning motivation of students. The attention, satisfaction, and confidence factors of motivation were significantly enhanced.

Another interesting mHealth application along with a computational model is presented in D. Baretta et al.'s paper. They present an experimental system formed by a mobile application, a wearable device, and a computational model that is conceptually framed in self-efficacy theory with a particular emphasis on self-efficacy beliefs and goal setting constructs. The computational model represents a mathematical description of a behaviour change model based on self-efficacy theory that needs to be tuned according to real case studies. The proposal of the authors turns out to be an innovative approach to promote physical activity behaviour change among inactive adults.

\section{Conflicts of Interest}

The guest editors declare that the work was conducted in the absence of any commercial or financial relationships that could be construed as a potential conflict of interest.

\section{Authors' Contributions}

All the guest editors wrote and contributed to and approved the final editorial.

Renato Ferrero

Maurizio Rebaudengo

Francisca Rosique 


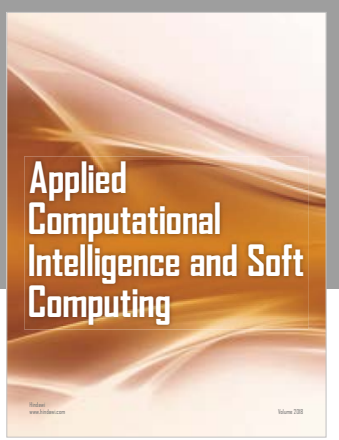

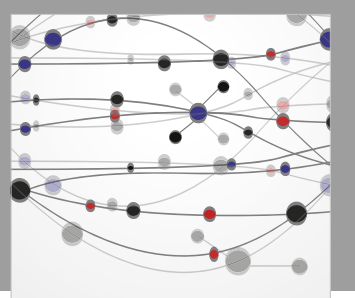

The Scientific World Journal
Submit your manuscripts at

Computing
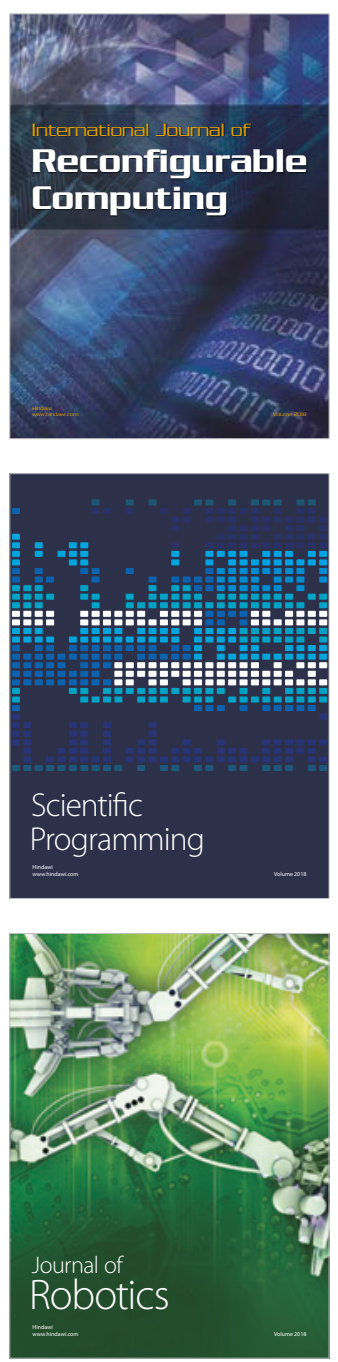

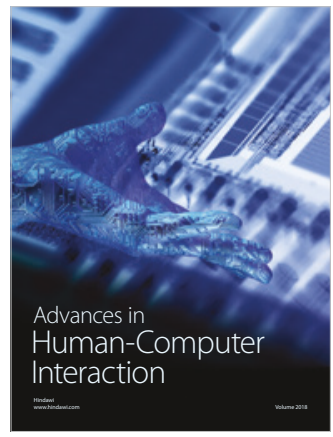

Human-Compute

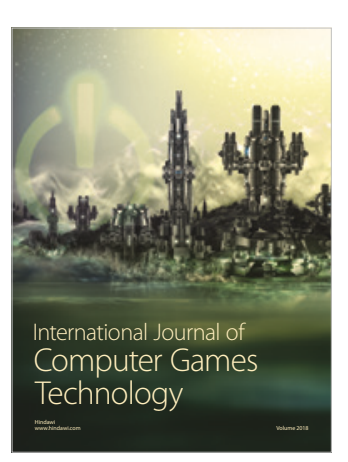

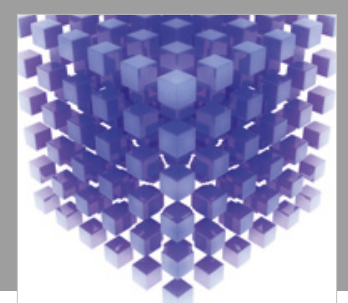

Mathematical Problems in Engineering

\section{Engincering}
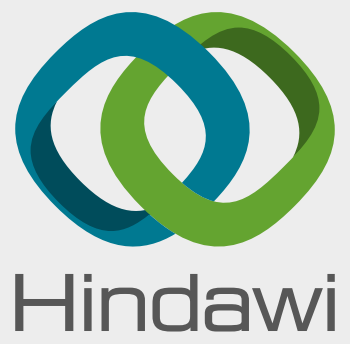

www.hindawi.com
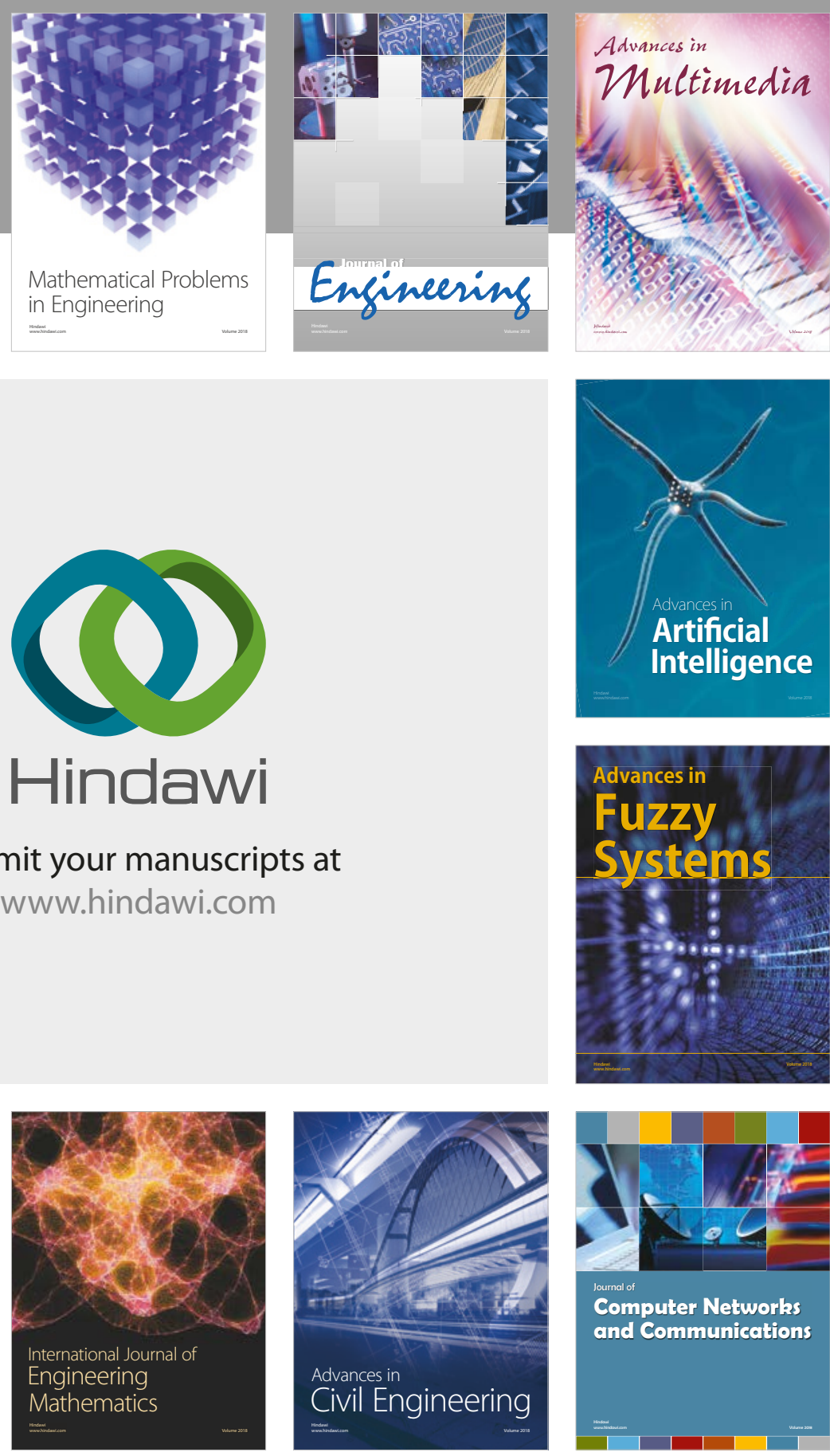

Computer Networks and Communications

Multimedia
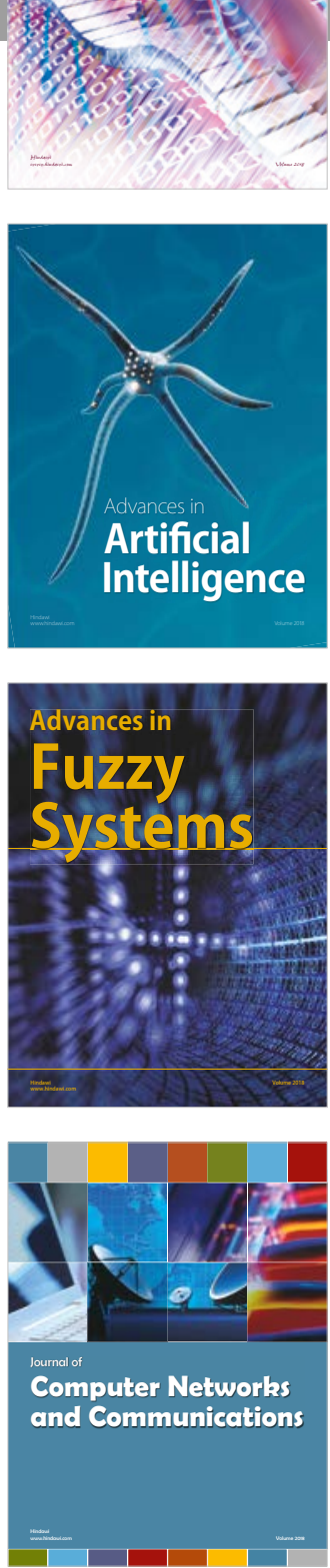

Advances in

Modelling \&

Simulation

in Engineering

interaction

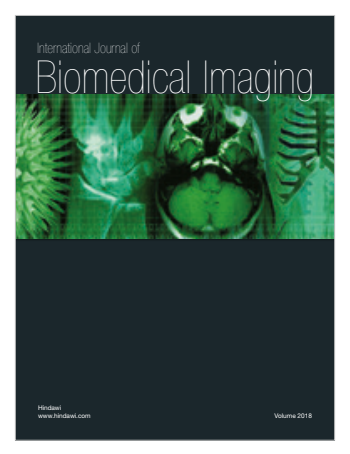

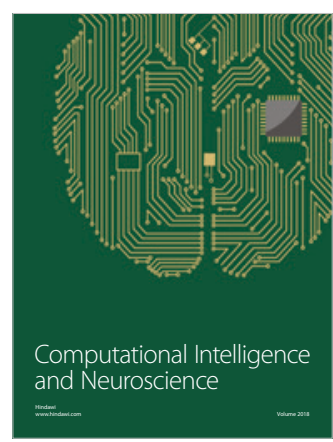

